\title{
Higher response rates in patients with severe chronic skin graft-versus-host disease treated with extracorporeal photopheresis
}

\author{
GABRIEL AFRAM ${ }^{1,2^{*},}$ EMMA WATZ ${ }^{3,4^{*}}$, MATS REMBERGER ${ }^{5,6}$, ULLA AXDORPH NYGELL $^{1,3,4}$, \\ MIKAEL SUNDIN ${ }^{7,8}$, HANS HÄGGLUND ${ }^{9}$, JONAS MATTSSON ${ }^{5,6^{*}}$, MICHAEL UHLIN ${ }^{3,4^{*}}$ \\ *The authors contributed equally to this work. \\ ${ }^{1}$ Hematology Centre, Karolinska University Laboratory, Stockholm, Sweden \\ ${ }^{2}$ Division of Hematology, Department of Medicine, Karolinska Institutet, Stockholm, Sweden \\ ${ }^{3}$ Department of Clinical Immunology and Transfusion Medicine, Karolinska University Laboratory, Stockholm, Sweden \\ ${ }^{4}$ Department of Clinical Science, Intervention and Technology, Division of Transplantation Surgery, Karolinska Institutet, \\ Karolinska Institutet, Stockholm, Sweden \\ ${ }^{5}$ Centre for Allogeneic Stem Cell Transplantation, Karolinska University Hospital, Stockholm, Sweden \\ ${ }^{6}$ Department of Oncology-Pathology, Karolinska Institutet, Stockholm, Sweden \\ ${ }^{7}$ Hematology/Immunology/SCT Section, Astrid Lindgren Children's Hospital, Karolinska University Hospital, Stockholm, Sweden \\ ${ }^{8}$ Division of Pediatrics, Department of Clinical Science, Intervention and Technology, Karolinska Institutet, Stockholm, Sweden \\ ${ }^{9}$ Department of Hematology, Uppsala University Hospital, Uppsala, Sweden
}

\begin{abstract}
Introduction: Different forms of graft-versus-host disease (GVHD) remain a major cause of morbidity and mortality after allogeneic hematopoietic stem cell transplantation (HSCT). The prognosis for steroid-refractory chronic GVHD (cGVHD) remains poor. Our aim was to evaluate extracorporeal photopheresis (ECP) treatment in $c G V H D$ patients with different organ involvement to detect subgroups of patients with the best response.

Material and methods: Thirty-four patients who underwent HSCT and developed moderate $(n=7)$ or severe $(n=27)$ steroid-refractory or steroid-dependent cGVHD treated with ECP were included in the analysis. A matched cGVHD control patient group untreated with ECP was collected for comparison.

Results: Compared to the control group and the stable/progressive disease (SD/PD) patients, individuals with complete/partial remission have higher overall survival and lower transplant-related mortality. Furthermore, patients with complete and partial remission $(C R / P R)$ had significantly higher levels of albumin and platelets after ECP treatment compared to patients with stable or progressive $c G V H D(S D / P D)$. Corticosteroid treatment and other immunosuppressive agents could successfully be tapered in the CR/PR group compared to the SD/PD patients. In this study patients with skin cGVHD are those with the highest rate of $C R / P R$ after ECP treatment.

Conclusions: Our results suggest that ECP treatment is safe and effective for patients with predominantly skin, oral and liver $c G V H D$.
\end{abstract}

Key words: $E C P, c G V H D$, treatment.

(Centr Eur J Immunol 2019; 44 (1): 84-91)

\section{Introduction}

Graft-versus-host disease (GVHD) remains a major cause of morbidity and mortality after allogeneic hematopoietic stem cell transplantation (HSCT). Improvements in immunosuppressive conditioning regimens have reduced the incidence and severity of acute GVHD, but the incidence of chronic GVHD (cGVHD) ranges from $20 \%$ to as high as $80 \%$ in different patient populations [1-5]. Chronic
GVHD is one of the most serious complications of HSCT, affecting both quality of life and mortality in long-term survivors. The impact on the individual patient depends on the severity and number of organs involved, which also allows classification of patients according to the National Institute of Health's (NIH) criteria into mild, moderate and severe cGVHD [6, 7]. Importantly, cGVHD is also associated with a strong graft-versus-leukemia effect, reducing the risk of malignant relapse after transplantation [8].

Correspondence: Gabriel Afram, Hematologiskt Centrum M54, Karolinska University Hospital, Huddinge SE-141 86 Stockholm, Sweden, e-mail: gabriel.afram@karolinska.se

Submitted: 27.01.2018; Accepted: 10.04.2018 
Risk factors for cGVHD include recipients of older age, prior acute GVHD, female donor-to-male recipient, HLA disparity between recipient and donor and use of peripheral blood as graft [9-11]. Approximately half of the patients respond to first-line treatment, mainly with corticosteroids given with or without a calcineurin inhibitor. The prognosis for steroid-refractory cGVHD remains poor $[12,13]$. Different treatment options are available, including extracorporeal photopheresis (ECP), but there are no clear strategies for individual patients. The "trial-anderror" approach remains the only way to identify the most appropriate regimen [14]. Therefore, treatment of cGVHD should be personalized, i.e. based on patient and disease characteristics.

Extracorporeal photopheresis has been used with varying degrees of success to treat steroid-refractory cGVHD since the late 1990s, and it is viewed as a well-tolerated intervention with steroid-sparing effect $[15,16]$. In the present study, ECP was used to treat a series of patients with moderate to severe cGVHD. The aim of this study was to retrospectively evaluate the effectiveness of ECP and to determine which subset of patients with cGVHD would benefit most from treatment.

\section{Material and methods}

The study was approved by the Regional Ethical Review Board in Stockholm (entry no. 425/97). Thirty-four patients who were treated with ECP in 1998-2011 due to moderate $(n=7)$ or severe $(n=27)$ steroid-refractory or steroid-dependent cGVHD after HSCT were included in this retrospective study. Patients that did not respond to primary immunosuppressive treatment strategies for cGVHD (corticosteroids and cyclosporine A - CsA) during this time period were either given ECP, mycophenolate mofetil (MMF) or other research-oriented treatments. The choice of these second-line modalities was based on the treating physician's choice. ECP was not chosen for those with ongoing infections, far distances between their homes and ECP clinic and for those patients who refused this treatment modality due to personal preference. All patients that received ECP were given the treatment as a second-line regimen following first-line immunosuppression described above.

In total, during this period, 881 patients underwent HSCT and 134 patients developed moderate to severe cGVHD. Chronic GVHD was retrospectively categorized according to the NIH consensus criteria [6]. Median age of the patient cohort was 32 (range 1.5-65) years, with 22 males and 12 females. Twenty-two patients received myeloablative conditioning and 12 received reduced-intensity conditioning. The majority of patients $(n=28)$ received peripheral mobilized stem cell grafts. After the patients were classified with cGVHD according to the NIH criteria, a representative age- and disease-matched control
Table 1. Characteristics of patients treated with extracorporeal photopheresis (ECP) due to severe chronic graftversus-host disease (GVHD) after hematopoietic stem cell transplantation (HSCT) and controls

\begin{tabular}{lccl}
\hline Characteristics & ECP & Controls & $\boldsymbol{p}$-value \\
\hline$n$ & 34 & 34 & \\
\hline Sex (male/female) & $22 / 12$ & $22 / 12$ & NS \\
\hline Age, years & $32(1.5-65)$ & $40(<1-62)$ & NS \\
\hline Children, $<18$ years & $13(38 \%)$ & $7(21 \%)$ & NS \\
\hline
\end{tabular}

Diagnosis

\begin{tabular}{lccc}
\hline Non-malignant & 2 & 2 & \\
\hline AML/ALL & $11 / 7$ & $7 / 7$ & NS \\
\hline Chronic leukemia & 3 & 6 & \\
\hline Lymphoma & 4 & 3 & \\
\hline MDS & 5 & 3 & \\
\hline Other malignancies & 2 & 6 & NS \\
\hline $\begin{array}{l}\text { Disease stage } \\
\text { (early/late) }\end{array}$ & $18 / 16$ & $14 / 20$ &
\end{tabular}

(early/late)

\begin{tabular}{lccc}
\hline Donor & \multicolumn{1}{l}{} & NS \\
\hline Sibling & 17 & 22 & \\
\hline MUD & 15 & 8 & \\
\hline Mismatched & 2 & 4 & \\
\hline Donor age, years & $36(0-66)$ & $41(0-61)$ & NS \\
\hline Female to male & $8(24 \%)$ & $8(24 \%)$ & NS \\
\hline SC source & $5 / 28 / 1$ & $4 / 28 / 2$ & NS \\
(BM/PBSC/CB) & & & \\
\hline TNC dose, $\times 10^{8} / 1$ & $12.0(0.5-28.3)$ & $10.0(0.4-28.3)$ & NS \\
\hline CD34+ dose, $\times 10^{6} / 1$ & $9.5(0.2-26.8)$ & $7.2(0.1-28)$ & NS \\
\hline Conditioning & & & \\
\hline MAC/RIC & $24 / 10$ & $18 / 16$ & NS \\
\hline TBI-based & 11 & 15 & NS \\
\hline Chemotherapy-based & 23 & 19 & \\
\hline ATG & $19(56 \%)$ & $15(44 \%)$ & NS \\
\hline GVHD prophylaxis & & & \\
\hline CsA + MTX & 32 & 27 & \\
\hline CsA + Prednisolone & 1 & & \\
\hline Tacrolimus + & & & \\
\hline
\end{tabular}

group was selected from the local HSCT quality registry based on similar cGVHD status. The controls and patients were matched based on the global NIH score, in which the controls had to have the same index organ severity as the patients. The characteristics of the patients and controls are summarized in Table 1. 


\section{Apheresis procedure}

All patients underwent apheresis using a Cobe Spectra system (Terumo BCT, Lakewood, CO, USA) with the AutoPBSC program via venous access through peripheral veins or, most commonly, a central venous catheter. Each time, an equivalent of one blood volume was processed. Anticoagulation was achieved with ACD-A at a ratio of $1: 11$ to $1: 13$.

\section{UV-A treatment}

The cells collected were diluted in saline solution $(0.09 \% \mathrm{NaCl})$ to a final volume of $300 \mathrm{ml}$. 8-methoxypsoralen (8-MOP) was then added to the cells before transfer to a UV-A-permeable EVA-bag (MacoPharma, Mouvaux, France). After hematocrit measurement to ensure a hematocrit of $<2 \%$, the cells were exposed to UV-A irradiation at $2 \mathrm{~J} / \mathrm{cm}^{2}$ (Vilber Lourmat, Marne-laVallée, France) $[17,18]$. The cells were then immediately re-infused to the patient. In children, the cell product volume was reduced before infusion.

\section{Extracorporeal photochemotherapy treatment regime}

Extracorporeal photochemotherapy (ECP) procedures were performed on two consecutive days every week until a clinical response was achieved, and then tapered by slowly extending the treatment intervals (every other week and eventually every four weeks). Treatments were discontinued either when a stable clinical response was reached or when there was treatment failure. Stable clinical response was defined as a partial or complete remission of cGVHD that was sustained over 3 months. The definition of stable clinical response also included patients with stable disease, that is, with no improvement or worsening of cGVHD during a 3-month period after ECP initiation. Treatment failure was defined as progressive disease, i.e. worsening of cGVHD during ECP treatment.

Table 2. Frequency of organ manifestations with relation to NIH organ specific score of 0-3. Most patients displayed more than one organ manifestation

\begin{tabular}{lcccc}
\hline \multicolumn{5}{c}{ NIH organ specific score } \\
\hline Organ & NIH 0 & NIH 1 & NIH 2 & NIH 3 \\
\hline Gastrointestinal & 14 & 7 & 10 & 3 \\
\hline Skin & 16 & 3 & 6 & 9 \\
\hline Liver & 16 & 6 & 6 & 6 \\
\hline Oral & 20 & 8 & 5 & 1 \\
\hline Pulmonary & 23 & 4 & 2 & 5 \\
\hline Joints and fascia & 27 & 6 & 1 & 0 \\
\hline Eyes & 28 & 6 & 0 & 0 \\
\hline
\end{tabular}

\section{Chronic graft-versus-host disease prophylaxis}

All patients except one received GVHD prophylaxis with cyclosporine A (CsA) or tacrolimus combined with methotrexate. The remaining patient received CsA and prednisone.

\section{Retrospective patient evaluation}

Chronic GVHD was originally prospectively diagnosed and documented according to the Seattle criteria [4]. For the current study, information was extracted from patient charts and reviewed to retrospectively assess the data according to the NIH classification. The assessment included the patient's medical history, histological samples, radiological and physiological examinations such as pulmonary function tests (PFT). PFT was conducted every three to six months during treatment and parameters such as $\mathrm{FEV}_{1}, \mathrm{FEV}_{1} / \mathrm{VC}$ (vital capacity) and diffusion capacity were collected.

In the current study, evaluation of ECP treatment was based on medical records and NIH cGVHD classification at set time points: 8 weeks and 6 months after initiation of ECP.

A complete response (CR) to ECP was defined as full resolution of cGVHD. If improvement in cGVHD was observed with a decrease of $\geq 1$ point on the organ-specific NIH cGVHD score, this was defined as a partial response (PR). The definition of stable disease (SD) included no change in cGVHD activity observed and progressive disease (PD) was defined as progressing cGVHD activity during or up to 8 weeks after cessation of ECP treatment. The results of laboratory measurements including hemoglobin levels, white blood cell (WBC) counts, platelet counts and albumin levels before and 8 weeks after cessation of ECP treatment were collected and analyzed. Alterations in laboratory parameters before ECP compared to 8 weeks after cessation were analyzed, and so was the difference between the two patient groups (CR/PR vs. $\mathrm{SD} / \mathrm{PD})$.

\section{Immunosuppressive treatment}

Most patients (80\%) had been on immunosuppressive treatment with prednisone $(1-2 \mathrm{mg} / \mathrm{kg})$ and CsA before starting ECP. The remaining $20 \%$ had different regimens including, among others, sirolimus, tacrolimus and MMF combined with prednisone. Response of cGVHD to firstline treatment was generally evaluated two to four weeks after initiation of therapy and subsequently every three months until cessation of treatment. All patients received antibacterial, antifungal and antiviral prophylaxis according to institutional guidelines.

\section{Chronic graft-versus-host disease}

Organ manifestations are described in Table 2. Most patients $(n=17)$ had cGVHD involving $1(n=9)$ or $2(n=8)$ organs. Furthermore, 8 patients had 3 organs involved, 4 patients had 4 organs involved and 5 had 5 to 7 organs involved. 
Skin cGVHD was seen in 18 patients: three with mild, six with moderate and nine with severe involvement. All patients with severe skin cGVHD had sclerodermatous presentation with erythematous rashes. One patient presented with a sentinel lesion in the form of a wound on both arms.

\section{Statistical analysis}

Overall survival (OS) was calculated using the Kaplan-Meier method and compared by the log-rank test. Transplant-related mortality (TRM) was estimated using a nonparametric estimator of cumulative incidence curves, taking competing events into consideration. Categorical parameters were compared using the $\chi^{2}$ test, and continuous variables were compared using the Mann-Whitney test. When comparing parameters before and after ECP the Wilcoxon matched pair test was used. Analysis was performed using the cmprsk software package (developed by Gray, June 2001), Splus 6.2 software (Insightful, Seattle, WA) and Statistica software (StatSoft, Tulsa, OK).

\section{Results}

\section{Patient outcome}

Overall survival for all 34 patients in this study was $82 \%$ at one year and $58 \%$ at 5 years after initiation of ECP. Median time to cGVHD onset was 200 (range 67-1222) days after HSCT. ECP was initiated within median 161 (range 10-1421) days after the onset of cGVHD.

\section{Clinical extracorporeal photochemotherapy evaluation}

The median number of ECP treatments was 22 (range 2-100) over median 26 (range 1-174) weeks. The ECP treatments were well tolerated and no side effects during cell infusions were reported.

After ECP treatment, $5 / 34$ patients $(15 \%)$ developed CR, 18 (53\%) PR and 8 patients (24\%) showed stable disease. Three patients $(9 \%)$ suffered from progress in disease during ECP.

\section{Organ-specific response rate}

Patients with CR/PR were predominantly those with cGVHD of the skin and oral mucosa with an organ-specific score (OSS) of 1-3 according to NIH classifications [6]. The most common organ involvement in partial responders was skin cGVHD with an OSS of 2-3 ( $n=13)$. The majority of such patients had a combination of skin and liver involvement, with an OSS for liver involvement of 2-3. Responses included softened skin and subcutaneous tissue, reduced erythema and decreased extent of scleroderma or hidebound skin. Isolated gastrointestinal (GI) or liver involvement (OSS 2-3) followed as the second largest group of responders.
Patients with GI, liver and pulmonary cGVHD were most frequent in the SD/PD group. In the patients with PD, the most common organ involvement was the lungs (OSS 2-3) (Table 3). The CR/PR group received almost twice as many ECP treatments [24 (7-100) vs. 14 (2-49), $p=0.09$ ] and for a four-times longer period of time than the SD/PD group [38 weeks (3-117) vs. 9 weeks (1-61), $p=0.02]$.

One CR/PR patient was treated for 117 weeks due to hidebound scleroderma with skin cGVHD grade 3 . The patient's condition improved with softening of the skin/subcutaneous tissue during the course of treatment, until the final 12 weeks in which the patient had developed SD with an OSS of 2. The SD/PD patient with 61 weeks of ECP treatment had a severe bronchiolitis obliterans and initially responded to ECP treatment when administered at a rate of one treatment every other week after which the treatment frequency was tapered. The patient rapidly developed more severe symptoms when the treatment was tapered to once a month, and finally died due to respiratory failure.

\section{Corticosteroid treatment}

In the data analysis stage of the current study we chose to analyze corticosteroid treatment before, at 8 weeks and 6 months after cessation of ECP treatment in order to obtain a more defined trend of sustained and decreased need of corticosteroids even after ECP treatment was stopped. There was a significant decrease in corticosteroid doses both early (+8 weeks) and late (+6 months) after ECP treatment (Fig. 1). In the CR/PR group, the corticosteroid dose was significantly lower 8 weeks after ECP than at the start $(p<0.001)$ and at 6 months after ECP a further decrease was seen $(p=0.02)$. In the SD/PD group, no significant decrease was detected.

\section{Laboratory measurements during extracorporeal photochemotherapy}

There was a significant increase in platelet counts and albumin levels after ECP treatment in CR/PR patients.

Table 3. Observed organ involvement of chronic graftversus-host disease and frequency of complete/partial response $(\mathrm{CR}+\mathrm{PR})$ versus stable disease/progressive disease $(\mathrm{SD} / \mathrm{PD})$ after extracorporeal photopheresis treatment for each organ site

\begin{tabular}{lcc}
\hline Organ manifestation & CR + PR & SD + P \\
\hline Gastro-intestinal & 12 & 8 \\
\hline Skin & 15 & 2 \\
\hline Liver & 12 & 6 \\
\hline Lungs & 7 & 6 \\
\hline Oral mucosa & 13 & 1 \\
\hline Joints and fascia & 6 & 1 \\
\hline Eyes & 5 & 1 \\
\hline
\end{tabular}




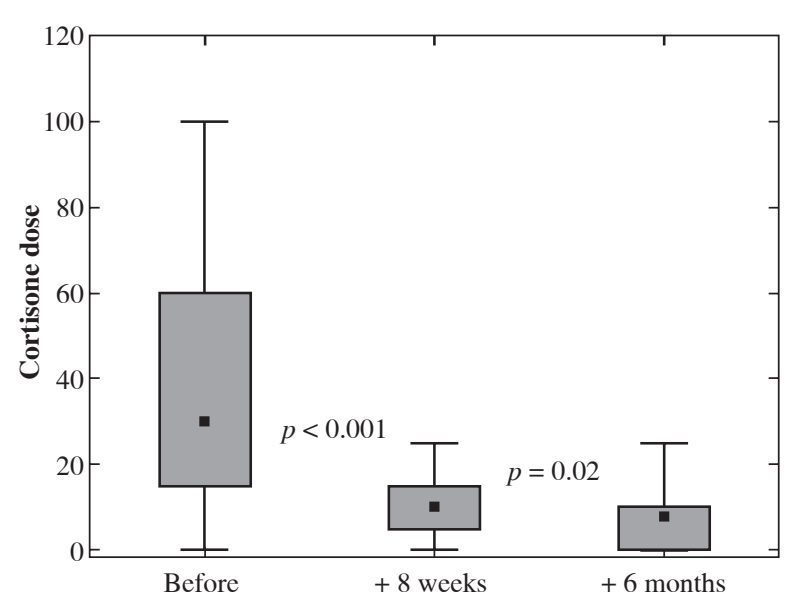

Fig. 1. Corticosteroid treatment shown as daily intake of milligrams of prednisone before the start of extracorporeal photopheresis (ECP) treatment, 8 weeks and 6 months after cessation of ECP
When studying the difference between the two patient groups (CR/PR vs. SD/PD) there were significant differences between the two patient groups with regard to both platelet and albumin levels before ECP (Fig. 2). In the $\mathrm{CR} / \mathrm{PR}$ group, both parameters were significantly higher after ECP treatment than in the group with SD/PD. WBC counts decreased in both $\mathrm{CR} / \mathrm{PR}$ and $\mathrm{SD} / \mathrm{PD}$, however the difference between the groups was not significant.

Hemoglobin levels remained unchanged after ECP treatment for both SD/PD and CR/PR.

\section{Cause of death}

Six patients died within a year after the start of ECP. The causes of death were bronchiolitis obliterans in three patients, pneumonia in one, invasive fungal infection in one and multiple organ failure in one patient. All of the aforementioned patients except one belonged to the group with SD/PD responses.
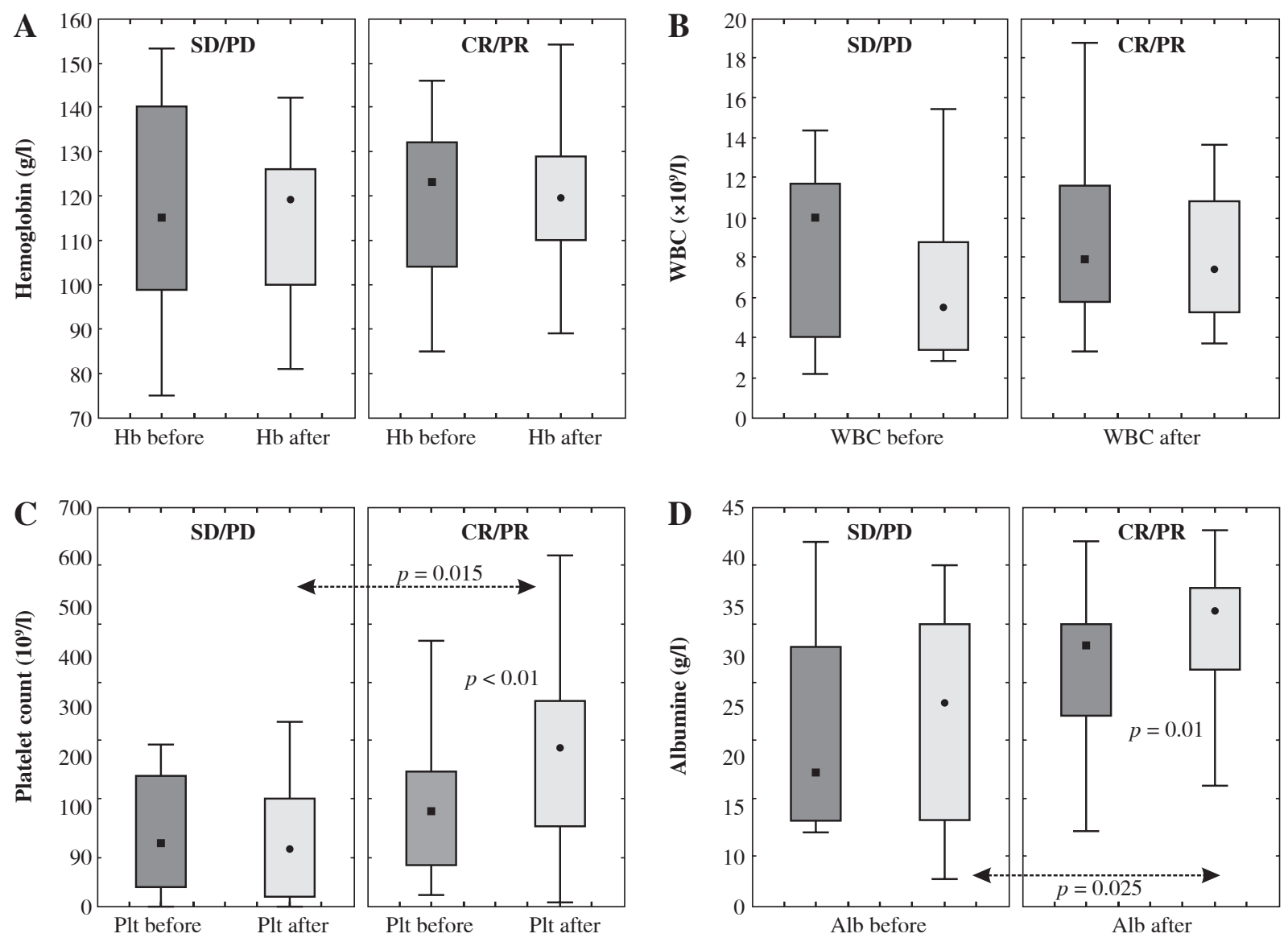

Fig. 2. Hemoglobin (Hb) levels, white blood cell counts (WBC), platelet (Plt) counts and albumin (Alb) levels before and after extracorporeal photopheresis treatment compared between patients with complete/partial remission (CR/PR) and those with stable/progressive disease (SD/PD) 


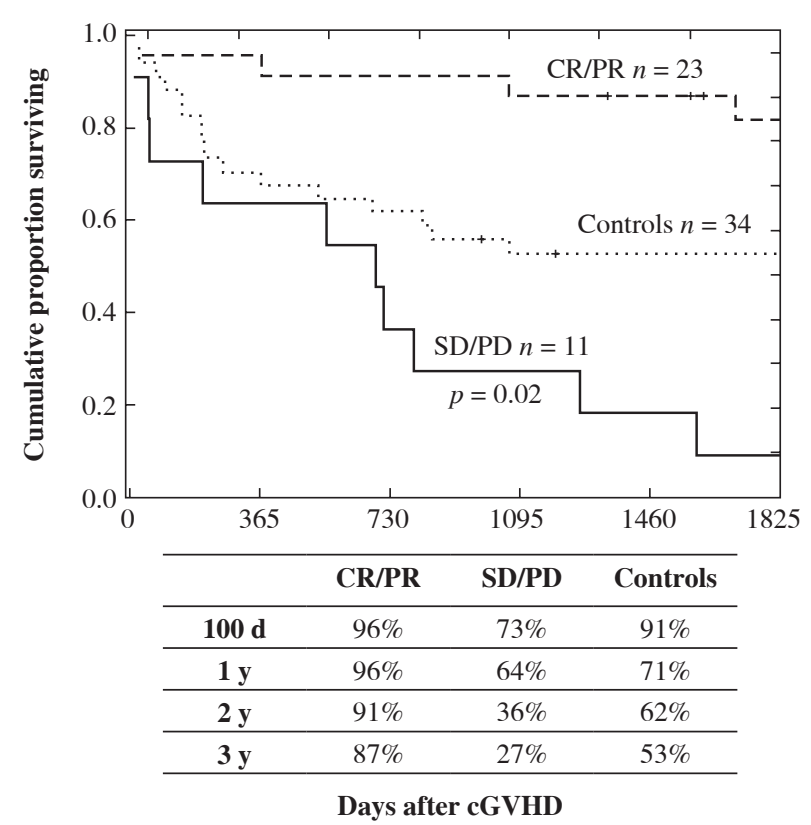

Fig. 3. Cumulative proportion of patients surviving at set time points after cGVHD diagnosis, compared between the $\mathrm{CR} / \mathrm{PR}, \mathrm{SD} / \mathrm{PD}$ and the control group

Eight additional patients died within five years after the start of ECP treatment. In two patients the cause of death was invasive fungal infection and two patients died from progressive bronchiolitis obliterans coinciding with invasive fungal infection. Other causes included pneumonia, gastrointestinal hemorrhage, septicemia and malignant relapse. Patients with $\mathrm{CR} / \mathrm{PR}$ had a higher survival rate compared to patients with SD/PD (Fig. 3).

\section{Clinical comparison to the control group}

As shown in Figure 3, the cumulative surviving proportion was higher in the $\mathrm{CR} / \mathrm{PR}$ patient group as compared to $\mathrm{SD} / \mathrm{PD}(p<0.001)$ and the controls $(p<0.01)$ (SD/PD vs. controls, $p=0.035)$. In addition, the lowest rate of TRM was seen in individuals with CR/PR (Fig. 4) (CR/PR vs. SD/PD, $p<0.001 ; \mathrm{SD} / \mathrm{PD}$ vs. controls, $p=0.001)$. The most predominant cause of death in the control group was relapse, followed by infection and cGVHD.

\section{Discussion}

Chronic GVHD remains a major cause of increased mortality and morbidity in long-term survivors after allogeneic HSCT. Second-line therapy for steroid-refractory or steroid-intolerant patients is not standardized. This study presents results concerning ECP as treatment for such patients.

ECP was first used successfully in the treatment of cutaneous T-cell lymphoma [19]. The mechanisms behind

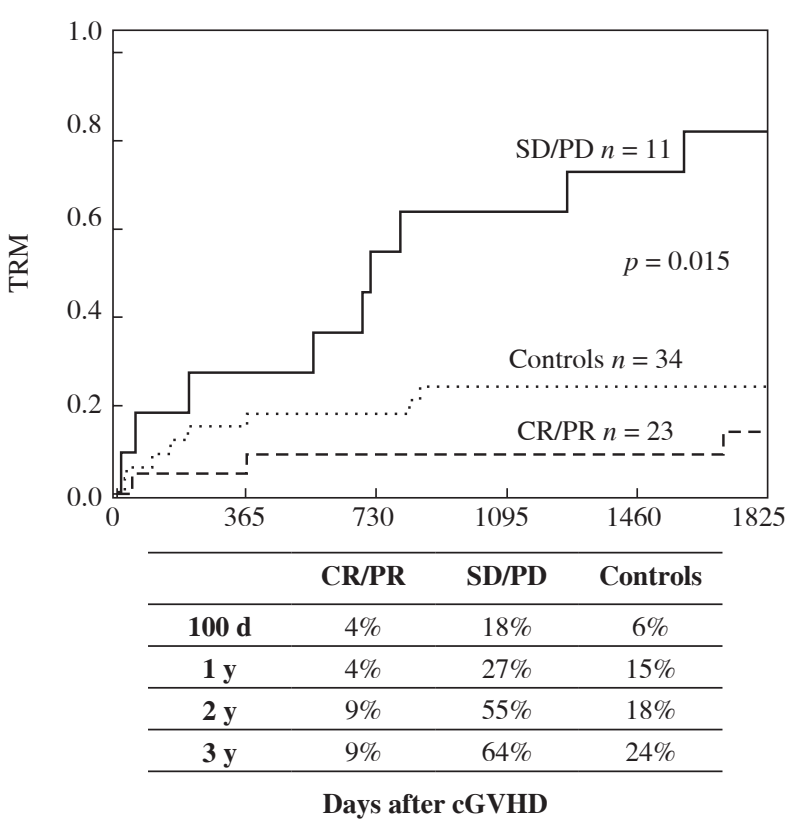

Fig. 4. Transplant-related mortality depicted over time from cGVHD diagnosis. Comparison between CR/PR, SD/ $\mathrm{PD}$ and control groups

the beneficial effects were uncertain at that time, although several studies have shed light on the matter. ECP induces apoptosis of all treated leukocytes within 24-48 hours of treatment. Antigen-presenting cells clear the infused ECP-treated cells (pre-apoptotic cells) which yields immune modulation with immune tolerance [18]. One mechanism behind this event is the upregulation of functionally active CD4+CD25+ FOXP3+ regulatory donor T-cells whose main role is to switch off autoreactive T-cells [20, 21].

The overall response rate $(0.5)$ in the current study is confirmed by a recent review in which Abu Dalle et al. showed an average response rate of 0.64 (95\% CI: 0.470.79 ) but with high variability between the described studies [20]. In the same review, complete responders were 0.26 (95\% CI: 0.05-0.55), which is in line with our results for the CR group with a response rate of 0.15 . The methodology for assessment of ECP response varies between different studies but most use organ-specific measurements [22, 23].

In line with previous studies, we also show that the highest response rate was seen in patients with skin cGVHD, the majority of these patients were only partial responders [20] (Table 3), more complete responders were found among patients with liver cGVHD. Only one patient had isolated liver involvement; the rest had concurrent skin and/or visceral involvement. To our knowledge, this has not been described previously. Several previous studies have reported the highest response rate in skin and/or GI cGVHD [15, 24-27] and responses increase when skin le- 
sions are treated early before development of scleroderma features. In the current study, none of the aforementioned patients were most prevalent in the CR group, which mostly consists of patients with liver cGVHD. Perhaps this is due to the fact that the liver is a highly immunologically active organ and one can speculate that the vast majority of ECP-treated cells would be found in the liver and spleen; accordingly, immune modulatory effects should be highest in these organs. Most patients with skin cGVHD in the current study had scleroderma before ECP treatment was started. This may have contributed to the low response rate in these patients. Also, main organ manifestation with liver involvement was more predominant in our study as compared to others [23, 27].

At our center, a corticosteroid together with a calcineurin inhibitor is the first-line treatment for patients with cGVHD. This results in prolonged periods of heavy immunosuppression and risk of side effects such as osteoporosis, Cushing syndrome and infections. Our results indicate a significant decrease in the need for corticosteroid both in the short and long term after ECP treatment (Fig. 1). Here, we show that the corticosteroid treatment was successfully tapered in a timely fashion and discontinued in patients who responded completely, which in itself is an important clinical endpoint.

Leukocyte counts decreased after ECP treatment which may be attributed to the apoptotic process involved in ECP and also the tapering of corticosteroid treatment (Fig. 2). Additionally, platelet count showed a significant increase in the group of responders (Fig. 2). A low platelet count has been shown to be a poor prognostic indicator for cGVHD and is associated with a lower response rate to ECP [25]. Our results indicate that directly or indirectly, ECP may induce increased and normalized platelet levels in ECP responders. Moreover, albumin levels increased significantly in the CR/PR group as compared to the SD/ PD group. One would expect this group to be dominated by the prevalence of GI-cGVHD and this was also the case in the current study. The second largest group of patients with SD/PD were those with liver cGVHD and this also corresponds well with lower albumin levels due to impaired albumin production during active cGVHD. The observed differences in platelet counts and albumin levels between the two groups after ECP treatment may be used prospectively as essential parameters in evaluating treatment response before more evident clinical changes manifest, e.g. skin softening, which can take up to a year to appear.

In the present study, the average length of treatment was greater in the CR/PR group than in the SD/PD group. Treatment was most likely discontinued in the SD/PD group due to ineffectiveness on cGVHD symptoms. As mentioned previously, six patients in the SD/PD group died within one year of treatment which also shortened treatment length in this group.
In addition, we showed that patients with CR/PR have higher survival and less TRM than the SD/PD group. Unexpectedly, when compared to the control group, the $\mathrm{CR} / \mathrm{PR}$ patients had better results in terms of TRM and survival. This would indicate a beneficial effect of ECP in terms of clinical outcome. To date these findings have been novel.

In conclusion, we found that patients with severe cGVHD involving primarily the skin followed by oral involvement, had the best response rates to ECP treatment. In this single center setting we can conclude that ECP is a safe treatment option for patients with steroid-refractory cGVHD. A good individual response against liver cGVHD was also noted which, to our knowledge, has not been reported before. We did not find convincing response rates in patients with severe pulmonary cGVHD, in contrast to a recent report [28]. Patients who responded to the ECP treatment had a significant increase in platelet and albumin levels. We think that laboratory parameters may have an important role as indicators of the clinical response to ongoing treatment. Our results suggest that ECP treatment is safe and effective for patients with skin, oral and liver cGVHD. We also show that patients meeting these criteria actually far better in terms of TRM and survival than those who do not receive ECP. In accordance with a recent study, we also show that ECP is a convincing option as second-line treatment for CGVHD and furthermore we define certain cGVHD organ involvement as better responders [29]. Further prospective trials are needed to determine whether treatment should be provided pre-emptively to certain patients who are at risk of developing severe cGVHD.

\section{The authors declare co conflict of interest.}

\section{References}

1. Thomas E (1975): Bone marrow transplantation (first of two parts). N Engl J Med 17: 832-843.

2. Bacigalupo A, Lamparelli T, Bruzzi P, et al. (2001): Antithymocyte globulin for graft-versus-host disease prophylaxis in transplants from unrelated donors: 2 randomized studies from Gruppo Italiano Trapianti Midollo Osseo (GITMO). Blood 98: 2942-2947.

3. Lee $\mathrm{S}$ (2005): New approaches for preventing and treating chronic graft-versus-host disease. Blood 2005; 105: 42004206.

4. Shulman HM, Sullivan KM, Weiden PL, et al. (1980): Chronicgraft-versus-host syndrome in man: a long-term clinicopathologic study of 20 Seattle patients. Am J Med 69: 204-217.

5. Thomas E (1975): Bone-marrow transplantation (second of two parts). N Engl J Med 24: 895-902.

6. Filipovich AH, Weisdorf D, Pavletic S, et al. (2005): National Institutes of Health Consensus Development project on Criteria for Clinical Trials in Chronic Graft-versus-Host Disease: I. Diagnosis and Staging Working Group report. Biol Blood Marrow Transplant 11: 945-956. 
7. Jagasia MH, Greinix HT, Arora M, et al. (2015): National Institutes of Health Consensus Development Project on Criteria for Clinical Trials in Chronic Graft-versus-Host Disease: I. The 2014 Diagnosis and Staging Working Group Report. Biol Blood Marrow Transplant 21: 389-401.e1.

8. Storb R, Gyurkocza B, Storer BE, et al. (2013): Graft-versushost disease and graft-versus-tumor effects after allogeneic hematopoietic cell transplantation. J Clin Oncol 31: 15301538 .

9. Ratanatharathorn V, Ayash L, Lazarus HM, et al. (2001): Chronic graft-versus-host disease: clinical manifestation and therapy. Bone Marrow Transplant 28: 121-129.

10. Atkinson K, Horowitz MM, Gale RP, et al. (1990): Risk factors for chronic graft-versus-host disease after HLA-identical sibling bone marrow transplantation. Blood 75: 2459-2464.

11. Eapen M, Logan BR, Confer DL, et al. (2007): Peripheral blood grafts from unrelated donors are associated with increased acute and chronic graft-versus-host disease without improved survival. Biol Blood Marrow Transplant 13: 14611468.

12. Pavletic SZ, Smith LM, Bishop MR, et al. (2005): Prognostic factors of chronic graft-versus-host disease after allogeneic stem cell transplantation. Am J Hematol 78: 265-274.

13. Akpek G, Zahurak ML, Piantadosi S, et al. (2001): Development of a prognostic model for grading chronic graft-versushost disease. Blood 97: 1219-1226.

14. Klingenberg C, Dawson JA, Gerber A, et al. (2011): Sustained inflations: comparing three neonatal resuscitation devices. Neonatology 100: 78-84.

15. Greinix HT, Volc-Platzer B, Rabitsch W, et al. (1998): Successful use of extracorporeal photochemotherapy in the treatment of severe acute and chronic graft-versus-host disease. Blood 92: 3098-3104.

16. Flowers ME, Apperley JF, van Besien K, et al. (2008): A multicenter prospective phase 2 randomized study of extracorporeal photopheresis for treatment of chronic graft-versus-host disease. Blood 112: 2667-2674.

17. Maeda A, Schwarz A, Kernebeck K, et al. (2005): Intravenous infusion of syngeneic apoptotic cells by photopheresis induces antigen-specific regulatory T cells. J Immunol 174: 5968-5976.

18. Peritt D (2006): Potential mechanisms of photopheresis in hematopoietic Stem cell transplantation. Biol Blood Marrow Transplant 12: 7-12.

19. Edelson R, Berger C, Gasparro F, et al. (1987): Treatment of cutaneos T-cell lymphoma by Extracorporeal photochemotherapy. N Engl J Med 316: 297-303.

20. Abu-Dalle I, Reljic T, Nishihori T, et al. (2014): Extracorporeal photopheresis in steroid-refractory acute or chronic graft-versus-host disease: results of a systematic review of prospective studies. Biol Blood Marrow Transplant 20: 16771686.

21. Seaton ED, Szydlo RM, Kanfer E, et al. (2003): Influence of extracorporeal photopheresis on clinical and laboratory parameters in chronic graft-versus-host disease and analysis of predictors of response. Blood 102: 1217-1223.

22. Foss FM1, DiVenuti GM, Chin K, et al. (2005): Prospective study of extracorporeal photopheresis in steroid refractory or steroid-resistant extensive chronic graft-versus-host disease: analysis of response and survival incorporating prognostic factors. Bone Marrow Transplant 35: 1187-1193.

23. Garban F, Drillat P, Makowski C, et al. (2005): Extracorporeal chemophototherapy for the treatment of graft-versus-host disease: hematologic consequences of short-term, intensive courses. Haematologica 90: 1096-1101.

24. Couriel DR, Hosing C, Saliba R, et al. (2006): Extracorporeal photochemotherapy for the treatment of steroid-resistant chronic GVHD. Blood 107: 3074-3080.

25. Greinix HT, Worel N, Just U, Knobler R (2014): Extracorporeal photopheresis in acute and chronic graft-versus-host disease. Transfus Apher Sci 50: 349-357.

26. Dignan FL, Greenblatt D, Cox M, et al. (2012): Efficacy of bimonthly extracorporeal photopheresis in refractory chronic mucocutaneous GVHD. Bone Marrow Transplant 47: 824830.

27. Hautmann AH, Wolff D, Hahn J, et al. (2013): Extracorporeal photopheresis in 62 patients with acute and chronic GVHD: Results of treatment with the COBE Spectra System. Bone Marrow Transplant 48: 439-445.

28. Del Fante C and Perrotti C. (2017): Extracorporeal photopheresis for bronchiolitis obliterans syndrome after allogeneic stem cell transplant: An emerging therapeutic approach? Transphus Apher Society 56: 17-19.

29. Sakillari I, Gavrillaki E, Batsis I, et al. (2018). Favourable impact of extracorporeal photopheresis in acute and chronic graft versus host disease: Prospective single-center study. J Clin Apher 33: 654-550. 